\title{
Game-Based Channel Selection for UAV Services in Mobile Edge Computing
}

\author{
Y. Chen $\mathbb{D}^{1},{ }^{1}$ H. Xing, ${ }^{1}$ S. Chen, ${ }^{1}$ N. Zhang, ${ }^{2}$ X. Chen $\mathbb{D},{ }^{1}$ and J. Huang $\mathbb{D}^{3}$ \\ ${ }^{1}$ School of Computer Science, Beijing Information Science and Technology University, Beijing 100101, China \\ ${ }^{2}$ University of Windsor, Windsor, Canada \\ ${ }^{3}$ Beijing Key Laboratory of Petroleum Data Mining, China University of Petroleum, Beijing 102249, China \\ Correspondence should be addressed to Y. Chen; chenying@bistu.edu.cn
}

Received 22 October 2021; Revised 8 December 2021; Accepted 21 December 2021; Published 3 February 2022

Academic Editor: Xiaolong Xu

Copyright $\odot 2022$ Y. Chen et al. This is an open access article distributed under the Creative Commons Attribution License, which permits unrestricted use, distribution, and reproduction in any medium, provided the original work is properly cited.

\begin{abstract}
Computation offloading is a hot research topic in mobile edge computing (MEC). Computation offloading among multiedge nodes in heterogeneous networks can help reduce offloading cost. In addition, the unmanned aerial vehicles (UAVs) play a key role in MEC, where UAVs in the air communicate with ground base stations to improve the network performance. However, limited channel resources can lead to the increase of transmission delay and the decline of communication quality. Effective channel selection mechanisms can help address those issues by improving transmission rate and ensuring communication quality. In this paper, we study channel selection during communication between multiple UAVs and base stations in an MEC system with heterogeneous networks. To maximize the transmission rate of each UAV user, we formulate a channel selection problem and model it as a noncooperative game. Then, we prove the existence of Nash equilibrium (NE). In addition, we design a multiple UAV-enabled transmission channel selection (UTCS) algorithm to obtain the equilibrium strategy profile of all the UAV users. Experimental results validate that UTCS algorithm can converge after a finite number of iterations and it outperforms random transmission algorithm (RTA) and sequential transmission algorithm (STA).
\end{abstract}

\section{Introduction}

With the development of mobile computing, the number of mobile users has soared. However, the network resources are limited. To reduce the communication and computing delay, MEC is proposed as a promising paradigm [1,2]. The computing and storage capacities are provided at edge nodes, providing services for terminal tasks and effectively reducing data processing delay and energy consumption of terminal devices. 5G-enabled MEC [3] is composed of heterogeneous base stations, where small cell base stations (SBSs) [4] reuse the channel resources with macro cell base station (MBS). Users of MBS and SBSs use the same set of communication resources for data transmission, which can effectively improve the utilization of communication resources.

With the development of unmanned aerial vehicles (UAVs) technologies, smaller and cheaper UAVs are available. As such, UAVs are no longer only used in specific fields (such as military domain) but are also introduced into civil networks [5]. Due to the high altitude flight characteristics, UAVs have been used as a key component of MEC [6], providing aerial network services $[7,8]$ in the event of a disaster [9]. In the face of some major natural disasters (such as earthquakes, floods, and typhoons), deployed ground base stations are likely to be damaged and local network disruptions can occur. UAVs play a vitally important role in damaged network rescue. Due to the flexible operating altitude and wide coverage, UAVs can communicate with user terminals in network damaged areas to obtain computationally intensive tasks and then communicate with base stations in network normal areas for processing. In addition, with the help of UAVs' unique LoS link, UAVs can ensure high-quality communication in extreme environments $[10,11]$ (e.g., rain, snow, and broken trees). 
As the number of computing tasks brought by UAVs increases, the demand for communication resources keeps growing. However, channel resources for data transmission are still heavily limited. When the number of users is too large, the channel is multiplexed by deploying micro base stations. In this case, channel multiplexing would bring the issue of interference, resulting in a decrease in the transmission rate. In order to improve the data transmission rate, it is necessary to design a reasonable channel selection strategy to reduce the interference of data transmission. An effective channel selection strategy can improve the utilization of channel resources and ensure the high transmission rate of each computing task.

During network rescue, UAVs can communicate with user terminals and base stations. Many existing studies merely focused on the communication between UAVs and users, and the communication between multiple UAVs and ground base stations had not been explored widely. Thus, we study the communication between UAVs and ground base stations. UAVs transmit computing data to the aerial and ground integrated wireless networks and process the task with the help of the computing resources [12-14] of the ground base stations. As the number of tasks increases, the transmission rate [15] of each task decreases. That is mainly because of the interference caused by channel multiplexing. Considering the characteristics of tasks, each computing task [16] selects the appropriate channel for data transmission, which can effectively reduce channel multiplexing. We aim to propose a multi-UAV transmission channel selection method to achieve distributed and high-quality data transmission.

In this paper, aiming at the offloading problem in edge computing, we study channel selection strategies for communication between multi-UAV users and base stations in MEC with heterogeneous networks. The following are our main contributions:

(i) We consider the MEC with heterogeneous networks consisting of an MBS and multiple SBSs, in which each SBS is assigned an MEC server to provide computing resources for relevant users. In addition, the UAV users of SBSs use channel resources of the MBS for data transmission. We formulate a noncooperative game to model and analyze the multiUAV channel selection problem in such a heterogeneous network. In particular, our model considers the interference generated by channel multiplexing to ensure high-quality data transmission for each UAV user.

(ii) The channel selection strategies of users are coupled to each other, so it is difficult to optimize each user's data transmission rate simultaneously. To show the existence of Nash equilibrium (NE) in the formulated noncooperative game, we prove its equivalence to an exact potential game which has at least one NE.

(iii) To reach the NE of the formulated noncooperative game, we propose a multiple UAV-enabled transmission channel selection (UTCS) algorithm. The proposed algorithm operates in a completely distributed manner; that is, each user does not know the selection strategies of other users and independently adjusts the channel selection strategy according to their prior experience.

(iv) We perform extensive numerical simulations to validate UTCS algorithm to compute the NE solution (i.e., the equilibrium channel selection and the equilibrium data transmission rate of each user). Experimental results show that UTCS algorithm can converge quickly through a finite number of iterations. Compared with the random transmission algorithm (RTA) and sequential transmission algorithm (STA), UTCS algorithm can obtain NE channel selection strategy and each user can achieve a higher data transmission rate.

The remainder of this paper is organized as follows. In Section 2, we briefly summarize the related researches. We model the communication between UAV users and base stations based on heterogeneous network scenarios and establish a noncooperative game model in Section 3. In Section 4, we analyze the existence of NE and design UTCS algorithm to obtain the equilibrium strategy. In addition, we illustrate the performance of UTCS algorithm through parameter analysis, convergence analysis, and comparison experiments in Section 5 and conclude our research work in Section 6.

\section{Related Work}

Some researches have been done on UAV network [17-22]. Zhang et al. [19] considered two communication modes, $\mathrm{UAV}$ to base station and UAV to UAV, and divided the problem into three subproblems to optimize channel allocation and UAV speed, respectively. Gu et al. [20] considered the network scenario of multi-UAV collaborative work in the context of environment awareness and studied resource allocation and task scheduling of multi-UAV collaborative work based on reinforcement learning. Berate et al. [21] regarded the UAV as an aerial base station and proposed a user cache framework involving multiple UAVs. Then, QoE was used as an indicator to study the user cache for multiple UAV base station deployment and the optimal caching strategy was obtained. Zhao et al. [22] considered a network scenario in which UAV and base station (BS) cooperate to serve ground users and investigated the problem of transmission rate optimization through joint optimization of UAV trajectory and NOMA precoding. The above studies introduced UAVs into different network scenarios to optimize the data transmission process from different perspectives of network communication. We introduce UAVs into 5G heterogeneous networks, which can greatly improve the power efficiency and spectrum efficiency.

Channel selection is a hot issue in mobile network communication. Gour et al. [23] selected underlay D2D 
network and proposed D2D channel allocation and power allocation schemes with and without quality of service constraints and transformed the problem into a nonconvex mixed-integer nonlinear programming problem. Shattal et al. [24] focused on a new vehicle-mounted ad hoc network (VANETs) architecture in which nodes continuously and autonomously selected one of three channel selection strategies and applied evolutionary games to solve the channel selection problem. Ko et al. [25] studied the joint optimization problem of LTE channel selection and frame scheduling to maximize LTE throughput and proposed heuristic algorithms to solve the problem. However, these works do not consider the cochannel transmission interference factor, which leads to the result that channel selection is not accurate enough. Thus, considering the transmission interference in the same channel, we study the channel selection decision problem for multi-UAV users.

Game theory is a good tool to solve the problem of multiplayer competitive decision-making. Cui et al. [26] considered the dynamics and uncertainties in the environment for modeling, constructed the long-term resource allocation problem as a stochastic game to maximize the expected return, and applied the reinforcement learning theory to design algorithm to solve the problem. In order to solve the optimization problem of relay selection in UAV network, Liu et al. [27] proposed a matching game classification method based on the competitive relationship between players and constructed a basic preliminary model of UAV relay model. However, these works do not highlight the competitive relationship between users. In this paper, we consider the communication resource competition in the process of data transmission by multi-UAV users, and a noncooperative game model is established to describe the channel resource competition in multi-UAV communication.

This paper considers a heterogeneous network of multiple types of multiple base stations communicating with multiple UAV users. Since different base station users select the same channel for transmission and cause interference, we apply the noncooperative game method to construct the communication model between users and base stations. In order to maximize the transmission rate of each user, a scheme of user equilibrium channel selection strategy is presented. Then, we prove the existence of equilibrium strategy. Combining the strategy selection probability, we design UTCS algorithm to ensure that multi-UAV users can obtain their own equilibrium strategy in the $5 \mathrm{G}$ heterogeneous networks. Finally, the convergence and effectiveness of UTCS algorithm are verified by experiments.

\section{The UAV-Enabled Heterogeneous Network Model for MEC and the Game Problem Formulation}

3.1. UAV-Enabled Heterogeneous Network for MEC. We consider a UAV-enabled heterogeneous network as shown in Figure 1, where multiple SBSs are distributed in the macro cell. Each SBS owns an MEC server to provide computing resources for corresponding users. In this network, the UAV
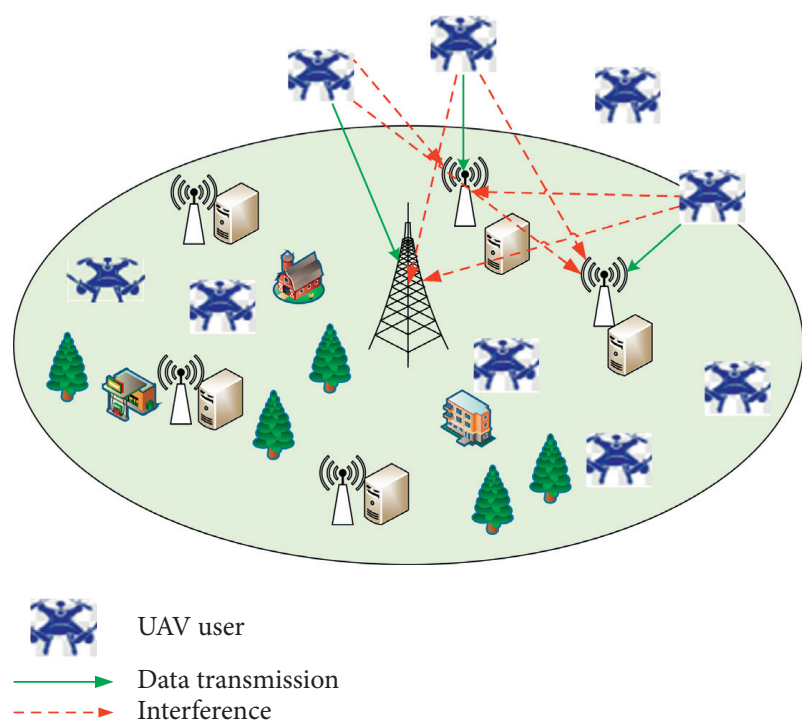

FIgURE 1: The UAV-enabled heterogeneous networks in MEC.

user transmits the data to the base station for task computation. The set of UAV users is defined as $\mathcal{N}=\{1,2, \ldots, N\}$, which can be further divided into macro cell base station users (MUs) and small cell base station users (SUs). Specifically, the MU is the user outside of the service area of the SBS and is served by MBS, and the set of MUs is defined as $\mathscr{N}_{0}=\left\{1,2, \ldots, N_{0}\right\}$. In addition, the set of SUs is represented by $\mathcal{N}_{M}=\left\{1,2, \ldots, N_{M}\right\}$. The set of all the base stations is represented as $\mathscr{M}=\mathscr{M}_{0} \cup \mathscr{M}_{m}=\{0,1, \ldots, M\}$, which contains an MBS and $M$ SBSs. The MBS provides services to MUs and SBSs provide services to SUs in their own service areas. $\mathscr{M}_{0}=\{0\}$ represents the MBS, which provides computing services to all the MUs. The set of SBSs is $\mathscr{M}_{m}=\{1,2, \ldots, M\}$, which is deployed in the service area of the MBS. The SUs' set of the SBS $m$ service area is $\mathcal{N}_{m}=\left\{1,2, \ldots, N_{m}\right\}$, and $\sum_{m \in \mathscr{M}_{m}}\left|\mathcal{N}_{m}\right|=\left|\mathcal{N}_{M}\right|$. The main symbols are given in Table 1 .

We define the channel set of the MBS as $\mathcal{S}=\{1,2, \ldots, S\}$. SBSs are deployed in the service area macro cell and the channels of the MBS are reused for data transmission. In addition, with dense deployment of SBS, it is considered that the number of users in each base station is no more than the number of channels, and users within the same base station do not need to reuse channels.

Due to the multiplexing of channels, there is interference when the user transmits the data, including the interference from MUs to SUs and the interference from SUs to SUs. Interference affects the data transmission rate, and users obtain efficient data transmission strategies by the analysis of the interference. $d_{i}$ denotes the channel selection strategy of user $i$, and $d_{i} \in \mathcal{S}$. The channel selection strategy profile of all the users is represented as $D=\left\{d_{1}, d_{2}, \ldots, d_{N}\right\}$. In addition, the users in the same base station do not reuse the channels; that is, $d_{i} \neq d_{j}, \forall i, j \in \mathcal{N}_{0}$, and $d_{i} \neq d_{j}, \forall i, j \in \mathcal{N}_{m}, m \in \mathscr{M}_{m}$.

When data is transmitted from UAV user to base station, the UAV is in a hover state and $U_{i}=\left[x_{i}, y_{i}, H_{i}\right]^{T}$ represents the location information of user $i$. The data transmission rate is affected by the channel gain which is related to the distance 
TABle 1: Notations.

\begin{tabular}{lc}
\hline Symbol & Description \\
\hline $\mathcal{N}$ & The set of UAV users \\
$\mathcal{N}_{0}$ & The set of MUs \\
$\mathcal{N}_{m}$ & The set of SUs \\
$\mathscr{M}$ & The set of base stations \\
$\mathscr{M}_{0}$ & The set of MBSs \\
$\mathscr{M}_{m}$ & The set of SBSs \\
$\mathcal{S}$ & The set of channels \\
$d_{i}$ & The channel selection strategy of user $i$ \\
$D$ & The channel selection strategy profile of all the users \\
$U_{i}$ & The location information of user $i$ \\
$Q_{0}$ & The location information of the MBS \\
$Q_{m}$ & The location information of SBS $m$ \\
$\beta_{0}$ & The channel power of MBS \\
$\alpha_{i 0}$ & The decay coefficient of MBS \\
$\beta_{m}$ & The channel power of SBS $m$ \\
$\alpha_{i m}$ & The fading coefficient of SBS $m$ \\
$p_{i}$ & The transmission power of user $i$ \\
$\sigma^{2}$ & The background noise \\
$d_{-i}$ & The channel selection strategy profile other than user $i$ \\
$B$ & The bandwidth of the base station
\end{tabular}

between the user and the base station. The position coordinate of MBS is defined as $Q_{0}=\left[x_{0}, y_{0}, H_{0}\right]^{T}$, and $Q_{m}=$ $\left[x_{m}, y_{m}, H_{m}\right]^{T}$ represents the position of SBS $m$.

Different from ordinary users on the ground, the UAV and the base station may transmit data through the LoS link [28]. The path loss for LoS link transmission $\alpha_{i n}^{L o S}$ and the path loss for NLoS link transmission $\alpha_{i n}^{N L O S}$ between UAV user $i$ and base station $n$ are expressed, respectively, as follows:

$$
\begin{aligned}
\alpha_{i n}^{\operatorname{LoS}} & =20 \log \left(\frac{4 \pi f_{c}\left\|Q_{n}-U_{i}\right\|}{c}\right)+\varepsilon^{\operatorname{LoS}}, \\
\alpha_{i n}^{\mathrm{NLoS}} & =20 \log \left(15 \frac{4 \pi f_{c}\left\|Q_{n}-U_{i}\right\|}{c}\right)+\varepsilon^{\mathrm{NLoS}},
\end{aligned}
$$

where $\varepsilon^{\text {LoS }}$ represents the average value of excessive path loss in LoS link and $\varepsilon^{N L O S}$ is the average value of excessive path loss in NLoS link. $f_{c}$ is the carrier frequency, $c$ is the speed of light, and $\left\|Q_{n}-U_{i}\right\|$ is the distance between UAV user $i$ and base station $n$. It is worth noting that base station $n$ is either an MSB or an SBS. The probability of data transmission between UAV user $i$ and base station $n$ through the LoS link is given as

$$
\rho_{\text {in }}=\frac{1}{1+b e^{-\varsigma\left(\arcsin \left(H_{n}-H_{i} /\left\|Q_{n}-U_{i}\right\|\right)-b\right)},}
$$

where $b$ and $\varsigma$ are environmental parameters determined by the deployment environment of UAVs. $\arcsin \left(H_{n}-H_{i} /\right.$ $\left.\left\|Q_{n}-U_{i}\right\|\right)$ is the horizontal angle between UAV user $i$ and base station $n$.

The average path loss between UAV user $i$ and base station $n$ is denoted as

$$
\bar{\alpha}_{\text {in }}=\rho_{\text {in }} \alpha_{\text {in }}^{\mathrm{LoS}}+\left(1-\rho_{\text {in }}\right) \alpha_{\text {in }}^{\mathrm{NLoS}},
$$

and the channel gain $h_{i}^{0}$ of MU $i$ can be calculated as follows:

$$
h_{i}^{0}=\frac{\beta_{0}}{\left\|Q_{0}-U_{i}\right\|^{\bar{\alpha}_{i 0}}}
$$

where $\left\|Q_{0}-U_{i}\right\|$ represents the distance between MU $i$ and MBS, and $\beta_{0}$ is the channel power of MBS.

The channel gain $h_{i}^{m}$ between SU $i$ and SBS $m$ is defined as

$$
h_{i}^{m}=\frac{\beta_{m}}{\left\|Q_{m}-U_{i}\right\|^{\bar{\alpha}_{i m}}},
$$

where $\left\|Q_{m}-U_{i}\right\|$ is the transmission distance between SU $i$ and SBS $m . \beta_{m}$ represents the channel power of SBS $m$.

Based on the channel gain and multiplexing, users can attain the channel selection strategy. We define $a_{i}^{s}$ to indicate whether user $i$ selects channel $s$ for data transmission. Specifically, when user $i$ selects channel $s$ for data transmission, $a_{i}^{s}=1$; otherwise, $a_{i}^{s}=0$. The SINR of MU $i$ is given as follows:

$$
r_{i}^{0}=\frac{a_{i}^{s} h_{i}^{o} p_{i}}{\sum_{m \in M_{m}} \sum_{j \in \mathcal{N}_{m}} h_{j}^{m} p_{j} a_{j}^{s}+\sigma^{2}},
$$

where $p_{i}$ and $p_{j}$ are the transmission powers of MU $i$ and SU $j$, respectively. $\sigma^{2}$ represents the noise power. $\sum_{m \in \mathscr{M}_{m}} \sum_{j \in \mathcal{N}_{m}} h_{j}^{m} p_{j} a_{j}^{s}$ represents the interference to MU $i$ by SU $j$ that selects the same channel for transmission as MU $i$.

For SUs, we calculate the interference between the MBS and the SBS and the interference between SBSs. The SINR of $\mathrm{SU} i$ is as follows:

$$
r_{i}^{m}=\frac{a_{i}^{s} h_{i}^{m} p_{i}}{\sum_{j \in \mathcal{N}_{o}} h_{j}^{0} p_{j} a_{j}^{s}+\sum_{m \in \mathscr{M}_{m}} \sum_{j \in \mathcal{N}_{m / j \neq i}} h_{j}^{m} p_{j} a_{j}^{s}+\sigma^{2}},
$$

where $\sum_{j \in \mathcal{N}_{o}} h_{j}^{0} p_{j} a_{j}^{s}$ denotes the interference of MU $j$ to SU $i$, and $\sum_{m \in \mathscr{M}_{m}} \sum_{j \in \mathcal{N}_{m}} h_{j}^{m} p_{j} a_{j}^{s}$ represents the interference of other SBS users to SU $i$.

Each user considers the impact of strategies for other users on themselves when obtaining channel selection strategy. We define the channel selection strategy profile of all the users other than user $i$ as follows:

$$
d_{-i}=\left\{d_{1}, d_{2}, \ldots, d_{i-1}, d_{i+1}, \ldots, d_{N}\right\} .
$$

When user $i$ selects channel $s$ for data transmission, the transmission rate of user $i$ can be given by

$$
R_{i}\left(d_{i}, d_{-i}\right)= \begin{cases}\frac{1}{N_{0}} B \log _{2}\left(1+r_{i}^{0}\right), & i \in \mathcal{N}_{o}, \\ \frac{1}{N_{m}} B \log _{2}\left(1+r_{i}^{m}\right), & i \in \mathcal{N}_{m},\end{cases}
$$

where $B$ is the bandwidth of the base station. The bandwidth of each base station is the same, and the base station allocates the bandwidth resource evenly to each user in its coverage. In addition, not only is the data transmission rate affected by 
the user's own channel selection strategy, but also it depends on other users' strategies.

3.2. The Game Problem Formulation. In this model, we regard UAV users as rational users. Each user is a selfish decision-maker, and they only care about their own benefits. Specifically, UAV users select the channel with the highest data transmission rate, considering their locations and channel reuse. Therefore, the objective function of user $i$ is given as follows:

$$
\max _{d_{i} \in \mathcal{S}} R_{i}\left(d_{i}, d_{-i}\right), \quad \forall i \in \mathcal{N} .
$$

Because of channel multiplexing, the channel selection strategies of users affect each other. If there are too many users selecting the same channel, interference will be more severe among these users, and the transmission rate of these users will decrease. Therefore, each user prefers to select the channel selected by a small number of users in pursuit of the maximum transmission rate. However, in real multiuser data transmission, users do not know the channel selection strategies of other users when making the transmission decision. Therefore, users are independent decision-makers and can only follow their prior experience to make decisions.

In the process of pursuing their own benefits maximization, users form a competitive relationship with each other. We can describe this process of user channel selection as a noncooperative game model. Then, the game can be formulated as follows:

$$
\Gamma=\left(\mathscr{N},\{\delta\}_{i \in \mathscr{N}},\left\{R_{i}\right\}_{i \in \mathcal{N}}\right),
$$

where $\mathcal{N}$ is the set of game players, which include both MUs and SUs. $\mathcal{S}$ is the feasible strategy profile of the participant and also is the channel set. Note that the set of feasible strategies is the same for each user. In addition, $R_{i}$ is the benefit of participant $i$ 's strategy, which is the transmission rate. The transmission rate of each user varies as the channel selection strategy changes.

In $\Gamma$, each user adjusts its channel selection strategy to gain more benefits. After a certain number of iterations, all the users reach a state in which they can no longer improve their benefits by changing their strategies. Therefore, all the users would keep the strategy unchanged. In this state, there is an equilibrium between all the users.

\section{Analysis of Nash Equilibrium and Decision Algorithm Design}

4.1. Proof of the Existence of NE. We consider an equilibrium state. Since users can no longer improve their own benefits by adjusting their strategies in this equilibrium state, the channel selection strategies of all users are no longer changed. The equilibrium state makes a tradeoff among all the users; that is, considering the satisfaction of all the users, the benefits of each user reach the relative best state. In other words, the equilibrium state satisfies the goal of maximizing the transmission rate of each user in $\Gamma$, which is called a Nash equilibrium (NE), and we give the definition of the $\mathrm{NE}$ of $\Gamma$ in the following.

Definition 1. For a noncooperative game $\Gamma$, if there is a channel selection strategy profile $D^{*}=\left\{d_{1}^{*}, d_{2}^{*}, \ldots, d_{N}^{*}\right\}$, and no user is willing to unilaterally change its channel selection strategy to improve its transmission rate in this environment, then

$$
R_{i}\left(d_{i}^{*}, d_{-i}^{*}\right) \geq R_{i}\left(d_{i}, d_{-i}^{*}\right), \quad \forall d_{i} \in \mathcal{S}, i \in \mathcal{N},
$$

and $D^{*}$ is the NE strategy profile of $\Gamma$.

When all the users select the equilibrium strategy for data transmission, they have no intention to further change the channel selection strategy because each user reaches the maximum benefit in the current environment. Therefore, $\mathrm{NE}$ is a stable state and a solution that satisfies the goal of $\Gamma$. In the following, we discuss the existence of NE.

The exact potential game has the finite improvement property (FIP), which indicates that the exact potential game can reach $\mathrm{NE}$ through a finite number of iterations. Therefore, we further prove the existence of $\mathrm{NE}$ in $\Gamma$ by proving that $\Gamma$ is an exact potential game. Then, we give the definition of the exact potential game.

Definition 2. For $\Gamma$, the channel selection strategy $d_{i}$ of user $i$, the channel selection strategy profile $d_{i}$ of all the users other than user $i$, and the objective function $R_{i}\left(d_{i}, d_{-i}\right)$ of $\Gamma$ are given. If and only if there is a potential function $\Phi\left(d_{i}, d_{-i}\right)$, the relationship between $\Phi\left(d_{i}, d_{-i}\right)$ and $R_{i}\left(d_{i}, d_{-i}\right)$ is as follows:

$$
R_{i}\left(d_{i}, d_{-i}\right)-R_{i}\left(d_{i}^{\prime}, d_{-i}\right)=\Phi\left(d_{i}, d_{-i}\right)-\Phi\left(d_{i}^{\prime}, d_{-i}\right),
$$

where $\Gamma$ is an exact potential game, and there is at least a pure strategy NE.

In $\Gamma$, we consider two types of users and they are independent of each other. Therefore, we prove the existence of NE for two kinds of users, respectively. In the following, we first analyze the case for MU.

Lemma 1. For $\Gamma$, if user $i$ is the $M U$, there is a function $\Phi^{0}(D)$ as follows:

$$
\Phi^{0}(D)=\frac{1}{N_{0}} B \log _{2}\left(\sum_{m \in \mathscr{M}_{m}} \sum_{j \in \mathcal{N}_{m}} h_{j}^{m} p_{j} a_{j}^{s}+h_{i}^{0} p_{i} a_{i}^{s}+\sigma^{2}\right),
$$

so that the relationship between $\Phi^{0}(D)$ and $R_{i}\left(d_{i}, d_{-i}\right)$ satisfies the following condition:

$$
R_{i}^{0}\left(d_{i}, d_{-i}\right)-R_{i}^{0}\left(d_{i}^{\prime}, d_{-i}\right)=\Phi^{0}\left(d_{i}, d_{-i}\right)-\Phi_{0}\left(d_{i}^{\prime}, d_{-i}\right) .
$$

Proof. When user $i$ is an MU, the utility function of MU $i$, that is, the transmission rate, can be given as 


$$
\begin{aligned}
R_{i}^{0}\left(d_{i}, d_{-i}\right)= & \frac{1}{N_{0}} B \log _{2}\left(1+\frac{a_{i}^{s} h_{i}^{o} p_{i}}{\sum_{m \in \mathscr{M}_{m}} \sum_{j \in \mathcal{N}_{m}} h_{j}^{m} p_{j} a_{j}^{s}+\sigma^{2}}\right) \\
= & \frac{1}{N_{0}} B \log _{2}\left(\frac{\sum_{m \in \mathscr{M}_{m}} \sum_{j \in \mathcal{N}_{m}} h_{j}^{m} p_{j} a_{j}^{s}+\sigma^{2}+a_{i}^{s} h_{i}^{o} p_{i}}{\sum_{m \in \mathscr{M}_{m}} \sum_{j \in \mathcal{N}_{m}} h_{j}^{m} p_{j} a_{j}^{s}+\sigma^{2}}\right) \\
= & \frac{1}{N_{0}} B \log _{2}\left(\sum_{m \in \mathscr{M}_{m}} \sum_{j \in \mathcal{N}_{m}} h_{j}^{m} p_{j} a_{j}^{s}+\sigma^{2}+a_{i}^{s} h_{i}^{o} p_{i}\right) \\
& -\frac{1}{N_{0}} B \log _{2}\left(\sum_{m \in \mathscr{M}_{m}} \sum_{j \in \mathcal{N}_{m}} h_{j}^{m} p_{j} a_{j}^{s}+\sigma^{2}\right) .
\end{aligned}
$$

We analyze the change of the utility function when the channel selection strategy of user $i$ changes. Specifically, when the transmission channel of user $i$ changes from channel $s$ to channel $s^{\prime}$, that is, from $a_{i}^{s}$ to $a_{i}^{s^{\prime}}$, the utility function changes as follows:

$$
\begin{aligned}
& R_{i}^{0}\left(d_{i}, d_{-i}\right)-R_{i}^{0}\left(d_{i}^{\prime}, d_{-i}\right) \\
&=\frac{1}{N_{0}} B \log _{2}\left(\sum_{m \in \mathscr{M}_{m}} \sum_{j \in \mathcal{N}_{m}} h_{j}^{m} p_{j} a_{j}^{s}+\sigma^{2}+a_{i}^{s} h_{i}^{o} p_{i}\right) \\
&-\frac{1}{N_{0}} B \log _{2}\left(\sum_{m \in \mathscr{M}_{m}} \sum_{j \in \mathscr{N}_{m}} h_{j}^{m} p_{j} a_{j}^{s}+\sigma^{2}\right) \\
&-\frac{1}{N_{0}} B \log _{2}\left(\sum_{m \in \mathscr{M}_{m}} \sum_{j \in \mathscr{N}_{m}} h_{j}^{m} p_{j} a_{j}^{s}+\sigma^{2}+a_{i}^{s^{\prime}} h_{i}^{o} p_{i}\right) \\
&+\frac{1}{N_{0}} B \log _{2}\left(\sum_{m \in \mathscr{M}_{m}} \sum_{j \in \mathcal{N}_{m}} h_{j}^{m} p_{j} a_{j}^{s}+\sigma^{2}\right) \\
&=\frac{1}{N_{0}} B \log _{2}\left(\sum_{m \in \mathscr{M}_{m}} \sum_{j \in \mathscr{N}_{m}} h_{j}^{m} p_{j} a_{j}^{s}+\sigma^{2}+a_{i}^{s} h_{i}^{o} p_{i}\right) \\
&-\frac{1}{N_{0}} B \log _{2}\left(\sum_{m \in \mathscr{M}_{m}} \sum_{j \in \mathscr{N}_{m}} h_{j}^{m} p_{j} a_{j}^{s}+\sigma^{2}+a_{i}^{s^{\prime}} h_{i}^{o} p_{i}\right)
\end{aligned}
$$

Then, we show that when the channel selection strategy of user $i$ changes, the potential function changes as follows:

$$
\Phi^{0}\left(d_{i}, d_{-i}\right)-\Phi^{0}\left(d_{i}^{\prime}, d_{-i}\right)
$$

$$
\begin{aligned}
= & \frac{1}{N_{0}} B \log _{2}\left(\sum_{m \in M_{m}} \sum_{j \in \mathcal{N}_{m}} h_{j}^{m} p_{j} a_{j}^{s}+\sigma^{2}+a_{i}^{s} h_{i}^{o} p_{i}\right) \\
& -\frac{1}{N_{0}} B \log _{2}\left(\sum_{m \in M_{m}} \sum_{j \in \mathcal{N}_{m}} h_{j}^{m} p_{j} a_{j}^{s}+\sigma^{2}+a_{i}^{s^{\prime}} h_{i}^{o} p_{i}\right) \\
= & R_{i}^{0}\left(d_{i}, d_{-i}\right)-R_{i}^{0}\left(d_{i}^{\prime}, d_{-i}\right) .
\end{aligned}
$$

Through the above proof, we can obtain Lemma 1.

Lemma 2. In $\Gamma$, when user $i$ is a small cell base station user, there is a potential function $\Phi^{m}(D)$ :

$$
\Phi^{m}(D)=\frac{1}{N_{m}} B \log _{2}\left(\sum_{j \in \mathcal{N}_{0}} h_{j}^{0} p_{j} a_{j}^{s}+\sum_{m \in \mathscr{M}_{m}} \sum_{j \in \mathcal{N}_{m}} h_{j}^{m} p_{j} a_{j}^{s}+\sigma^{2}\right) .
$$

When the channel selection strategy of user $i$ changes, the following condition is satisfied between $\Phi^{m}(D)$ and $R_{i}^{m}\left(d_{i}, d_{-i}\right)$ :

$$
R_{i}^{m}\left(d_{i}, d_{-i}\right)-R_{i}^{m}\left(d_{i}^{\prime}, d_{-i}\right)=\Phi_{m}\left(d_{i}, d_{-i}\right)-\Phi_{m}\left(d_{i}^{\prime}, d_{-i}\right) .
$$

Proof. If user $i$ is an SU, according to (9), the utility function of $\mathrm{SU} i$ is equivalent to 


$$
\begin{aligned}
R_{i}^{m}\left(d_{i}, d_{-i}\right)= & \frac{1}{N_{m}} B \log _{2}\left(1+\frac{a_{i}^{s} h_{i}^{m} p_{i}}{\xi^{0}+\sum_{m \in \mathscr{M}_{m}} \sum_{j \in \mathcal{N}_{m / j \neq i}} h_{j}^{m} p_{j} a_{j}^{s}+\sigma^{2}}\right) \\
= & \frac{1}{N_{m}} B \log _{2}\left(\frac{\xi^{0}+\sum_{m \in \mathscr{M}_{m}} \sum_{j \in \mathcal{N}_{m}} h_{j}^{m} p_{j} a_{j}^{s}+\sigma^{2}}{\xi^{0}+\sum_{m \in \mathscr{M}_{m}} \sum_{j \in \mathcal{N}_{m / j \neq i}} h_{j}^{m} p_{j} a_{j}^{s}+\sigma^{2}}\right) \\
= & \frac{1}{N_{m}} B \log _{2}\left(\xi^{0}+\sum_{m \in \mathscr{M}_{m}} \sum_{j \in \mathcal{N}_{m}} h_{j}^{m} p_{j} a_{j}^{s}+\sigma^{2}\right) \\
& -\frac{1}{N_{m}} B \log _{2}\left(\xi^{0}+\sum_{m \in \mathscr{M}_{m}} \sum_{j \in \mathscr{N}_{m / j \neq i}} h_{j}^{m} p_{j} a_{j}^{s}+\sigma^{2}\right),
\end{aligned}
$$

where $\xi^{0}=\sum_{j \in \mathcal{N}_{0}} h_{j}^{0} p_{j} a_{j}^{s}$ represents the interference of MUs to user $i$.
When the channel selection strategy of user $i$ changes, the utility function changes as follows:

$$
\begin{aligned}
R_{i}^{m}\left(d_{i}, d_{-i}\right)-R_{i}^{m}\left(d_{i}^{\prime}, d_{-i}\right)= & \frac{1}{N_{m}} B \log _{2}\left(\xi^{0}+\sum_{m \in \mathscr{M}_{m}} \sum_{j \in \mathcal{N}_{m}} h_{j}^{m} p_{j} a_{j}^{s}+\sigma^{2}\right) \\
& -\frac{1}{N_{m}} B \log _{2}\left(\xi^{0}+\sum_{m \in M_{m}} \sum_{j \in \mathcal{N}_{m / j \neq i}} h_{j}^{m} p_{j} a_{j}^{s}+\sigma^{2}\right) \\
& -\frac{1}{N_{m}} B \log _{2}\left(\xi^{0}+\sum_{m \in \mathscr{M}_{m}} \sum_{j \in \mathcal{N}_{m / j \neq i}} h_{j}^{m} p_{j} a_{j}^{s}+h_{i}^{m} p_{i} a_{i}^{s^{\prime}}+\sigma^{2}\right) \\
& +\frac{1}{N_{m}} B \log _{2}\left(\xi^{0}+\sum_{m \in M_{m}} \sum_{j \in \mathcal{N}_{m / j \neq i}} h_{j}^{m} p_{j} a_{j}^{s}+\sigma^{2}\right) \\
= & \frac{1}{N_{m}} B \log _{2}\left(\xi^{0}+\sum_{m \in \mathscr{M}_{m}} \sum_{j \in \mathcal{N}_{m}} h_{j}^{m} p_{j} a_{j}^{s}+\sigma^{2}\right)- \\
& \frac{1}{N_{m}} B \log _{2}\left(\xi^{0}+\sum_{m \in \mathscr{M}_{m}} \sum_{j \in \mathcal{N}_{m / j \neq i}} h_{j}^{m} p_{j} a_{j}^{s}+h_{i}^{m} p_{i} a_{i}^{s^{\prime}}+\sigma^{2}\right) .
\end{aligned}
$$

The relationship between the potential function and the utility function when the channel selection strategy changes is as follows:

$$
\begin{aligned}
\Phi^{m}\left(d_{i}, d_{-i}\right)-\Phi^{m}\left(d_{i}^{\prime}, d_{-i}\right) \\
=\frac{1}{N_{m}} B \log _{2}\left(\xi^{0}+\sum_{m \in \mathscr{M}_{m}} \sum_{j \in \mathcal{N}_{m}} h_{j}^{m} p_{j} a_{j}^{s}+\sigma^{2}\right) \\
\quad-\frac{1}{N_{m}} B \log _{2}\left(\xi^{0}+\sum_{m \in \mathscr{M}_{m}} \sum_{j \in \mathscr{N}_{m / j \neq i}} h_{j}^{m} p_{j} a_{j}^{s}+h_{i}^{m} p_{i} a_{i}^{s^{\prime}}+\sigma^{2}\right) \\
=R_{i}^{m}\left(d_{i}, d_{-i}\right)-R_{i}^{m}\left(d_{i}^{\prime}, d_{-i}\right) .
\end{aligned}
$$


We can obtain that when user $i$ is an SU, $\Gamma$ is an exact potential game. Thus, Lemma 2 is proved.

As shown above, we respectively prove the relationship between the potential function and the utility function of two types of users. Now we give the potential function of the whole $\Gamma$.

Theorem 1. $\Gamma$ is an exact potential game, and the potential function is as follows:

$$
\Phi(D)= \begin{cases}\Phi^{0}(D), & i \in \mathcal{N}_{o} \\ \Phi^{m}(D), & i \in \mathcal{N}_{m} .\end{cases}
$$

Proof. In $\Gamma$, we consider two types of users, MUs and SUs. In Lemmas 1 and 2, we theoretically prove whether the game is an exact potential game in two user cases. In addition, the two types of users are independent of each other. Specifically, a user will not be both an MU and an MU. Thus, the feasible strategy profiles of users do not affect each other. From Lemmas 1 and 2, we derive the following:

$R_{i}\left(d_{i}, d_{-i}\right)-R_{i}\left(d_{i}^{\prime}, d_{-i}\right)=\Phi\left(d_{i}, d_{-i}\right)-\Phi\left(d_{i}^{\prime}, d_{-i}\right), \forall i \in \mathcal{N}$.

According to the above proof, we conclude that, for each user $i \in \mathcal{N}$, when the channel selection strategy changes, the utility function and potential function satisfy the equality relation given in (25). Thus, $\Gamma$ is an exact potential game, and Theorem 1 is proved.

\subsection{Multiple UAV-Enabled Transmission Channel Decision} Algorithm (UTCD). Because the exact potential game has FIP property, users in $\Gamma$ can reach $N E$ after a finite number of iterations. We design the algorithm to reach the NE. Specifically, we consider how the user selects the transmission channel in each iteration. Designing a proper channel selection mechanism can make the game reach equilibrium quickly.

How to design effective methods to solve network game problems has been widely studied, for example, adopting the idea of best response. In the idea of best response, each user, in accordance with the principle of maximizing their own interests, continuously adjusts their strategies in each iteration to achieve equilibrium. Each user obtains the channel selection strategy by traversing its feasible strategy profile based on the current game environment and calculates the benefits respectively for comparison. It is a strategy iterative process. In each iteration, the user calculates the benefits of all the feasible strategies to obtain the most profitable strategy.

In the above solution process, the default strategies of different users can be the same. However, in the heterogeneous network, users of the same base station service cannot reuse the same channel for data transmission. Therefore, we introduce the channel selection probability and combine the channel selection probability with the best response [29] idea to design our channel selection algorithm. Considering the particularity of the scenario, we propose a channel strategy selection algorithm applicable to the UAV-enabled game model in Algorithm 1, that is, the multiple UAV-enabled transmission channel decision (UTCD) algorithm. Specifically, each user's feasible strategy profile is accompanied by a selection probability vector. For a user, the probability of selecting the feasible channel to transmit is different, and the change of probability is related to the corresponding benefits of the strategy. If the corresponding benefits of the selected strategy are higher, the selection probability of the strategy will be higher. However, the total probability for each user's feasible strategy profile is constant. The specific update method of the strategy selection probability is shown in Algorithm 2.

The probability of all users' strategy selection constitutes the strategy selection matrix, which is used to update the game environment. The selection of probability affects the benefits. When we update the game environment, we select the strategy with the maximal probability and at the same time ensure that users of the same base station service select different strategies. The main steps of UTCS algorithm are given as follows:

Strategy selection probability initialization. Each user's strategy profile corresponds to a selection probability vector. Therefore, the number of elements in the vector is equal to the number of elements in the strategy profile. The probability is initially evenly allocated to each strategy. The probability vector of initial strategy selection for user $i$ is given as follows:

$$
P_{i}(t=0)=\left(\frac{1}{S}, \frac{1}{S}, \ldots, \frac{1}{S}\right) .
$$

Game environment initialization. For users of a base station service, we assign the initial channel strategy to users in order of channel set $\mathcal{S}$. The initial channel selection for users of base station $m$ is $E_{m}(t=0)=\left\{1,2, \ldots, N_{m}\right\}, E_{m} \subseteq \mathcal{S}$. The initial channel selection of all the users constitutes the initial game environment.

Benefit calculation. In each iteration, the user adjusts the strategy selection probability by calculating the benefits. The benefits are calculated by (9), and the current game environment is considered in the calculation process. In addition, in order to satisfy the fairness and simultaneity of the user's decision, the user, after adjusting the probability vector, keeps the current strategy unchanged until the game environment updates.

Selection probability update. The selection probability is updated according to the calculated benefits of the selected strategy. The probability after the update is related to the current benefits and the probability before the update, and the specific calculation method is as follows:

$$
P_{i}(t+1)=P_{i}(t)+\varepsilon r_{i}^{t}\left(e_{d_{i}}^{t}-P_{i}(t)\right),
$$

where $\varepsilon$ is the update step size applied to control the overall change rate of the probability and $e_{d_{i}}^{t}$ is the unit 
(1) Initialize: $\mathcal{N}, \mathcal{S}, U_{i}, Q_{m}, Q_{0}, \beta_{0}, \beta_{m}, \alpha_{0}, \alpha_{m}, p_{i}, p_{j}, \sigma^{2}, a_{i}^{s}, B$;

(2) According to channel set $\mathcal{S}$, UAV users of the same base station service are allocated the initial transmission channel in order;

(3) The initial game environment is constituted as follows, $E(t=0)=\left(d_{1}, d_{2}, \ldots, d_{N}\right)$;

(4) The update strategy probability matrix of users is obtained from Algorithm 2 as follows, $\mathrm{E}(t)=\left(\begin{array}{cccc}p_{1}^{1} & \cdots & p_{1}^{S} \\ \vdots & & \vdots \\ p_{N}^{1} & \cdots & p_{N}^{S}\end{array}\right)$;

(5) According to $\mathrm{E}(t)$, the strategy with the highest probability of each UAV user $i$ is selected to form a new game environment.

(6) The selection method is, $d_{i}(t)=\arg \max P\left(d_{i}, d_{-i}\right), \forall d_{i} \in \mathcal{S}$;

(7) While satisfying the maximization principle, the selection of the channel selection strategy should ensure that there is no channel multiplexing between users of the same base station service;

(8) When all the users' selection strategies no longer change, the update is stopped;

Output: The equilibrium strategy profile, $D^{*}=\left\{d_{1}^{*}, d_{2}^{*}, \ldots, d_{N}^{*}\right\}$.

Algorithm 1: The multiple UAV-enabled transmission channel selection (UTCS) algorithm.

(1) The strategy selection probability of each MU $i$ is set according to the fairness principle, $P_{i}(t=0)=((1 / S),(1 / S), \ldots,(1 / S))$;

(2) for each MU $i \in \mathcal{N}$ do

(3) The transmission rate are calculated according to 10 ;

(4) Each UAV user's interference calculation is based on the current game environment;

(5) The following is applied to update the stratgy selection probability of user $i$; $P_{i}(t+1)=P_{i}(t)+\varepsilon r_{i}^{t}\left(e_{d_{i}}^{t}-P_{i}(t)\right)$

(6) end for

(7) The probability of strategy selection for all UAV users constitutes the strategy selection matrix;

Output: The update strategy probability matrix of users is, $\mathrm{E}(t)=\left(\begin{array}{ccc}p_{1}^{1} & \cdots & p_{1}^{S} \\ \vdots & & \vdots \\ p_{N}^{1} & \cdots & p_{N}^{S}\end{array}\right)$

Algorithm 2: The transmission strategy update algorithm.

vector whose $d_{i}$-th element is 1 . In addition, $r_{i}^{t}$ is the utility, which is obtained by $r_{i}^{t}=\eta_{i} R_{i}^{t}$, and $\eta_{i} \leq 1 / \max R_{i}$. After the selection probability of all the users has been updated, the next iteration is entered.

Game environment update. The user follows the principle of selecting the strategy of maximum probability; that is,

$$
d_{i}(t)=\arg \max P\left(d_{i}, d_{-i}\right), \quad \forall i \in \mathcal{N} .
$$

However, in the base station $m$, in order to avoid channel multiplexing, the users' strategy selections are different. Specifically, $N_{m}$ elements are selected with different rows and columns in the following probability matrix:

$$
\mathrm{E}_{m}=\left(\begin{array}{ccc}
p_{1}^{1} & \cdots & p_{1}^{S} \\
\vdots & & \vdots \\
p_{N_{m}}^{1} & \cdots & p_{N_{m}}^{S}
\end{array}\right)
$$

The probability of the strategy is generated according to the amount of the strategy benefits, and the greater the benefits are, the greater the probability increases.
Therefore, when the maximum selection probability strategies of different users are the same, the user strategy with the highest probability value is selected.

Termination. Each user selects a transmission strategy for each iteration. When the selections of all the users are no longer changed, the iteration ends, and the strategy profile is the desired equilibrium strategy profile as follows:

$$
D^{*}=\left\{d_{1}^{*}, d_{2}^{*}, \ldots, d_{N}^{*}\right\} .
$$

As shown above, all the users select strategies in the same game environment, which can ensure the synchronization of all the user decisions, and more in line with the reality.

\section{Performance Evaluation}

In this section, we first analyze the influence of the values of two parameters on the model and the convergence of the algorithm. Then, the performance of UTCS algorithm is analyzed by comparison experiment. 
We consider that there are $50 \mathrm{UAV}$ users in a UAV-enabled heterogeneous network with one MBS and 5 SBSs deployed. The user's location information is randomly generated within the service scope of the base station to which it belongs. For ease of calculation, we assume that all the UAVs hover at a fixed height of $20 \mathrm{~m}$. For the MBS, the altitude is $10 \mathrm{~m}$, and the altitude of SBSs is $5 \mathrm{~m}$. The power gain of the MBS is $-50 \mathrm{db}$, and the power gain of the SBSs is generated randomly from $[-30,-40] \mathrm{dB}$. In addition, the transmission power for each UAV user is randomly generated from $[100,500] \mathrm{mW}$. The bandwidth is $5 \mathrm{MHz}$, and the bandwidth is evenly distributed among users. The background noise is $-100 \mathrm{dbm}$. The initial values for the main parameters are set in Table 2.

5.1. Parameter Analysis. $\alpha$ is the path loss exponent in data transmission between the UAV user and the base station. We set different path loss exponents (i.e., 2.5, 2.6, and 2.7) to analyze the effect of path loss on the transmission rate. Figure 2 shows the impact of path loss exponent on channel gain at different transmission distances. We can see that, with the increase of distance, the overall transmission rate tends to decline, and the greater the distance is, the slower the transmission rate decreases. When the transmission distance is the same, the smaller $\alpha$ is, the higher the channel gain will be. In addition, Figure 3 shows the impact of path loss exponent on the channel gain with base stations of different channel power gains. Obviously, when the channel power gain increases, the channel gain tends to increase. The smaller the loss coefficient is, the higher the channel gain is. According to Figures 2 and 3, the higher the path loss is, the slower the channel gain is. The path loss index is determined by the actual environment in which the data is transmitted. Specifically, when the transmission environment is complex, the transmission loss will be high. Conversely, in a simple environment, the transmission loss is low; and the channel gain affects the transmission rate, so that the UAV hover location would be selected in a simple transmission environment to increase the transmission rate.

Figure 4 shows the impact of the update step size on the change rate of the selection probability of a strategy. $\varepsilon$ is the update step size of the selection probability for the strategy. As the transmission rate increases, the selection probability increases from 0.1. Moreover, the larger the update step size is, the faster the probability grows. In Figure 5, we show the effect of update step size on the convergence rate of transmission rate. As can be seen in the figure, with the increase of the number of iterations, the transmission rate of the UAV user gradually reaches a convergence state. The reason is that all users obtain the optimal transmission rate. Meanwhile, no user has the will to change strategy. The transmission rate's convergence is the fastest and the user's transmission rate is the largest when all situations have converged after 11 iterations, which is 0.6. Therefore, the selection of the appropriate update step size can affect the convergence rate and the user's equilibrium rate simultaneously.

5.2. Convergence Analysis. Figure 6 shows the convergence of user transmission strategies. Three users (i.e., user 9,
TABLE 2: Parameters used in the evaluation.

\begin{tabular}{lc}
\hline Parameters & Value \\
\hline The number of UAV users & 50 \\
The number of MBSs & 1 \\
The number of SBSs & 5 \\
The altitude of UAV users & $20 \mathrm{~m}$ \\
The altitude of the MBS & $10 \mathrm{~m}$ \\
The altitude of SBSs & $5 \mathrm{~m}$ \\
The power gain of the MBS & $-50 \mathrm{db}$ \\
The power gain of SBSs & {$[-30,-40] \mathrm{dB}$} \\
The transmission power for each UAV user & {$[100,500] \mathrm{mW}$} \\
The bandwidth & $5 \mathrm{MHz}$ \\
The background noise & $-100 \mathrm{dbm}$ \\
\hline
\end{tabular}

user 33, and user 39) are randomly selected to observe the trend of their channel selection strategies as the number of iterations increased. Theoretically, we prove the existence of NE in the game in Section 3. Therefore, it can be seen from Figure 6 that the channel selection strategies of these users show a convergence trend after a finite number of iterations. Specifically, user 9 converges after 4 iterations and finally selects channel 5 for data transmission. In the 10th iteration, the channel selection strategy of user 33 no longer changes. After 8 iterations, user 39 selects channel 6 for data transmission. Due to the different transmission power, channel gain, and other factors, different users finally reach the convergence of the strategy after different iterations.

When the user's channel selection strategy no longer changes, the user's transmission rate may not reach the constant state due to the influence of other users' adjustment strategies. Therefore, the convergence state of user's transmission rate can show the convergence rate more accurately. Figure 7 shows the convergence of user transmission rates. We randomly select user 2 , user 21 , and user 45 from 50 UAV users and analyze the change of user transmission rate as the number of iterations increases. As shown in Figure 7, with the increase of the number of iterations, the user's transmission rate gradually converges and eventually remains unchanged. Specifically, after 8 iterations of the UAV user, the user's transmission rate no longer changes. Due to channel multiplexing, there is interference between users. Therefore, the strategy changes of other users can affect the transmission rate of the user, and the transmission rates of all the users converge at the same time.

5.3. Comparison Analysis. Compared with other algorithms, we analyze the performance of UTCS algorithm. In the random transmission algorithm (RTA), each UAV user randomly selects a channel for data transmission. In the sequential transmission algorithm (STA), each user allocates channel resources in the order of the channel set. In UTCS algorithm, users adjust channel selection strategies with the goal of maximizing their own transmission rate and obtain the final channel selections through multiple iterations. Figure 8 shows the change in the total transmission rate for all the users as the number of iterations increases. Obviously, 


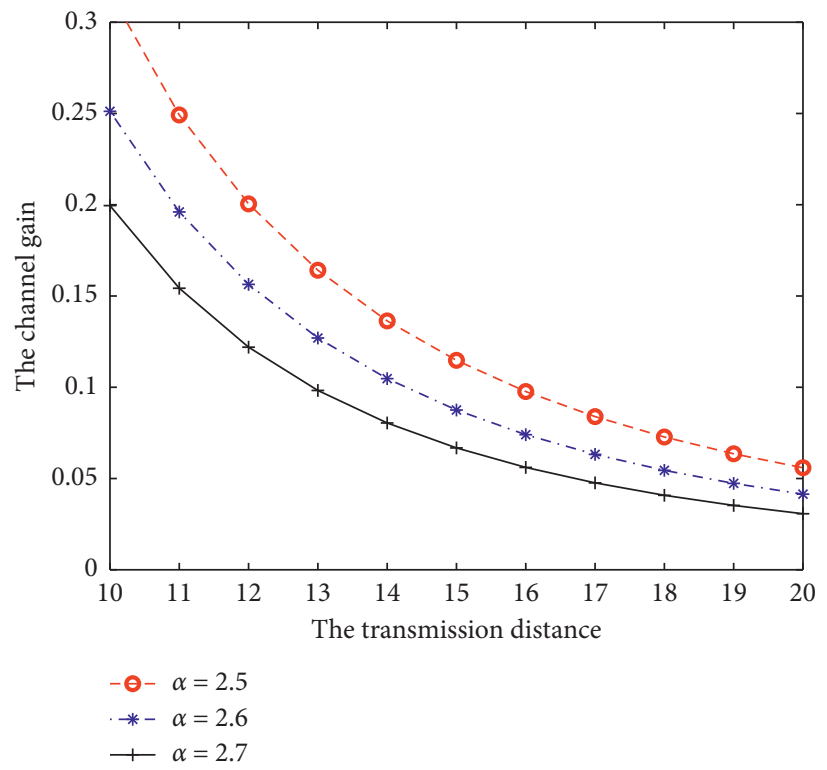

Figure 2: Impact of different values of the path loss exponent $\alpha$ with the change of transmission distance.

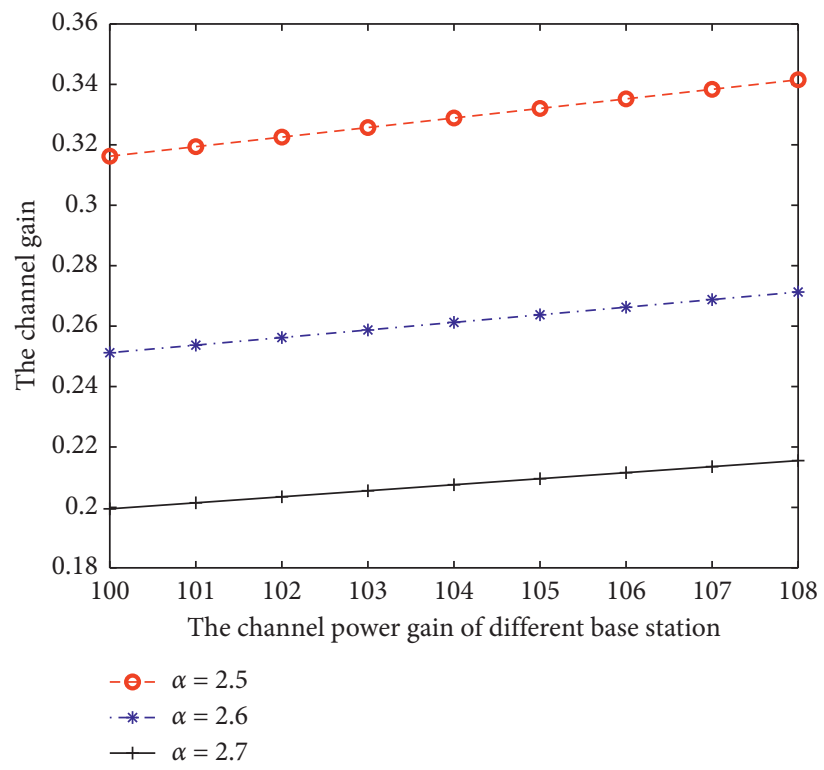

FIGURE 3: Impact of different values of the path loss exponent $\alpha$ with the change of power gain of different base station.

the total transmission rate of RTA is unstable due to its randomness, and the STA's fixed channel selection strategy keeps the total transmission rate unchanged. Notably, the transmission rate obtained by UTCS algorithm converges after 11 iterations. In the convergent state, we compare the total transmission rates obtained by three algorithms. Specifically, the total transmission rate obtained by UTCS algorithm is $2.15 \%$ higher than the maximum transmission rate of RTA and $4.3 \%$ higher than STA. It is worth noting that the random channel selection of RTA has a certain probability to make the total transmission rate reach the global optimal state; that is, the total transmission rate obtained by RTA is higher than that of UTCS algorithm. However, the probability of applying RTA to reach the global optimal transmission rate is very low and the global optimal state does not consider the maximum of each user's transmission rate, which does not conform to the goal of the model. Therefore, UTCS algorithm can perform the best.

In heterogeneous network, there is no channel multiplexing among MUs. Therefore, channel interference does not exist among MUs. The data transmission of SUs needs to reuse channel resources, so we analyze the impact of the number of SBSs deployed on the average transmission rate of users. In Figure 9, with the increase of the SBSs, the average transmission rate of users shows a downward trend after rising first. Because the number of MUs is less than the number of channels, channel resources are underutilized. When SUs reuse channel resources for data transmission, 


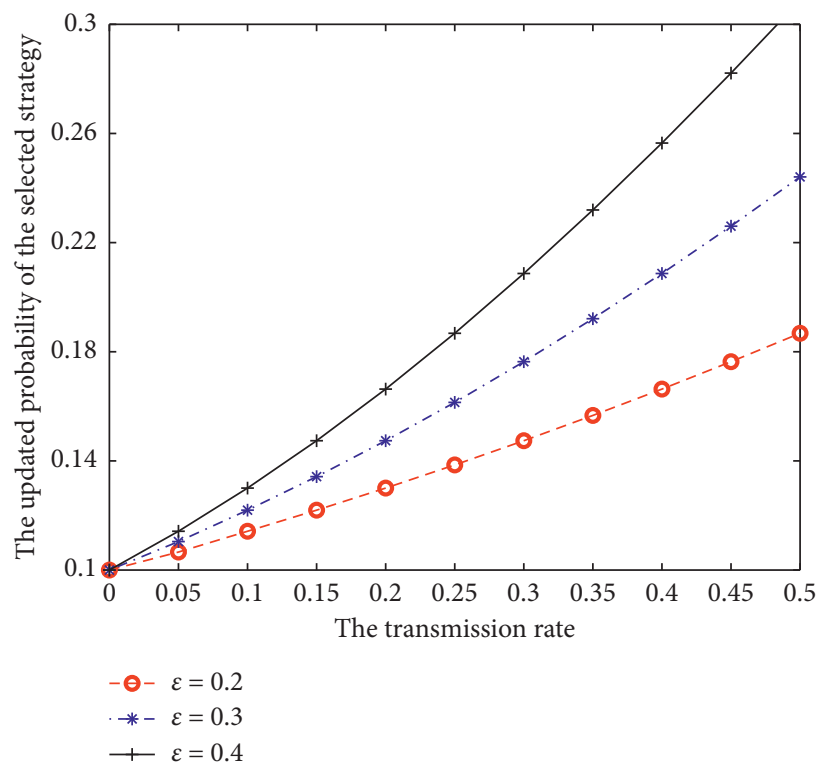

FIGURE 4: Impact of different values of the update step $\varepsilon$ on update rate of the selection probability.

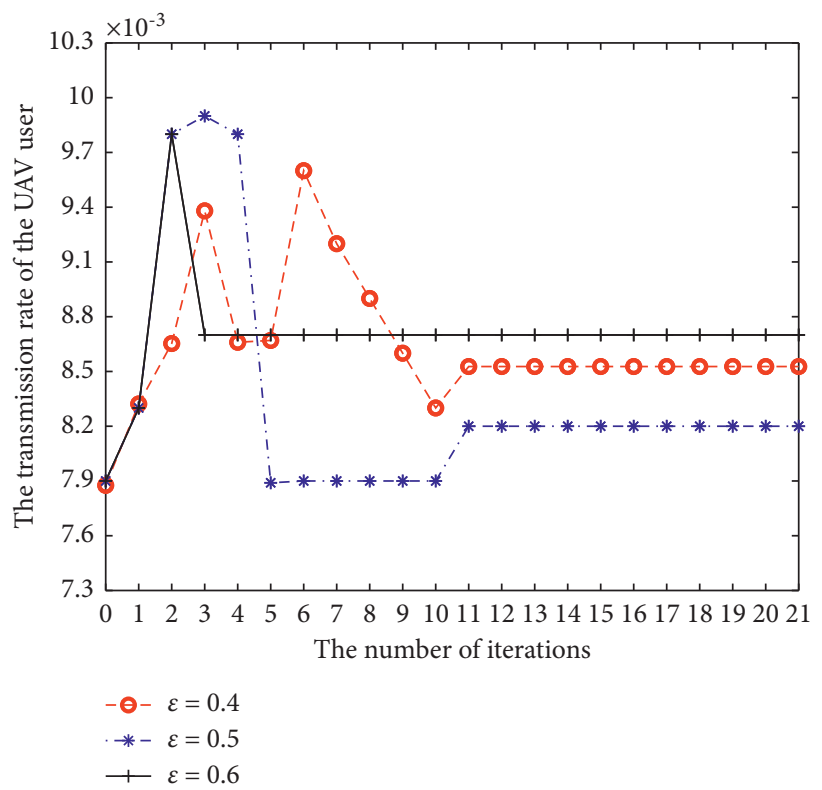

FIgURE 5: Impact of different values of the update step $\varepsilon$ on the convergence rate.

some channel resources are idle. Therefore, the interference is relatively low, and the average rate of users increases. In peak state, channel reaches saturation of resource utilization, and interference increases at a faster rate, so the average transmission rate of users goes down. When the number of
SBSs reaches 5 , the average transmission rate obtained by UTCS algorithm is $7.36 \%$ higher than that of RTA and $4.63 \%$ higher than that of STA. Therefore, UTCS algorithm can effectively reduce interference and slow down the rate of decline of the average transmission rate. 


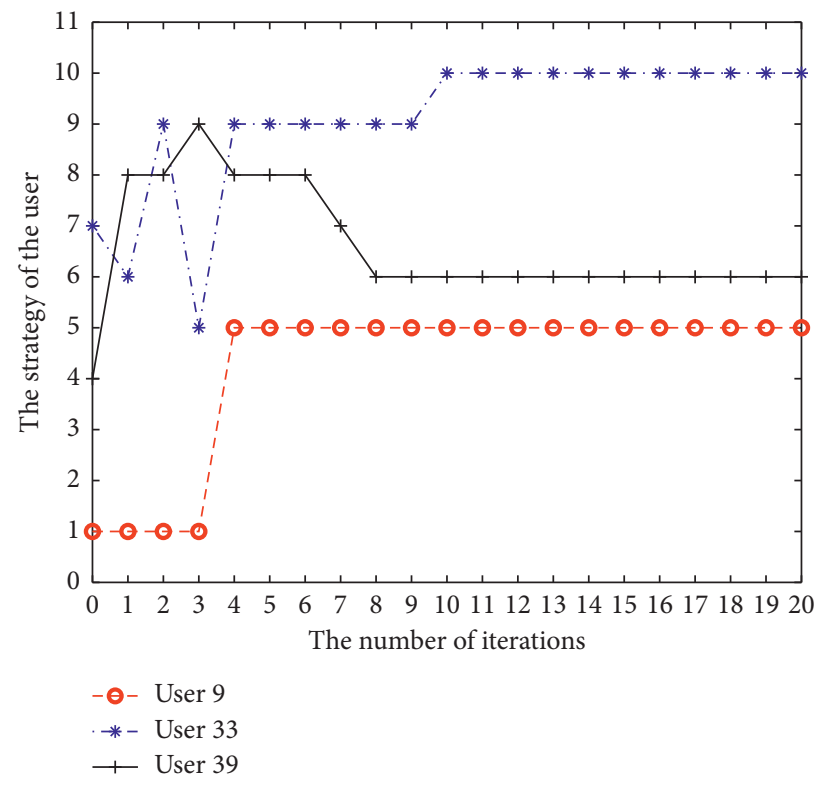

FIgURE 6: The convergence of channel selection strategy of the UAV users.

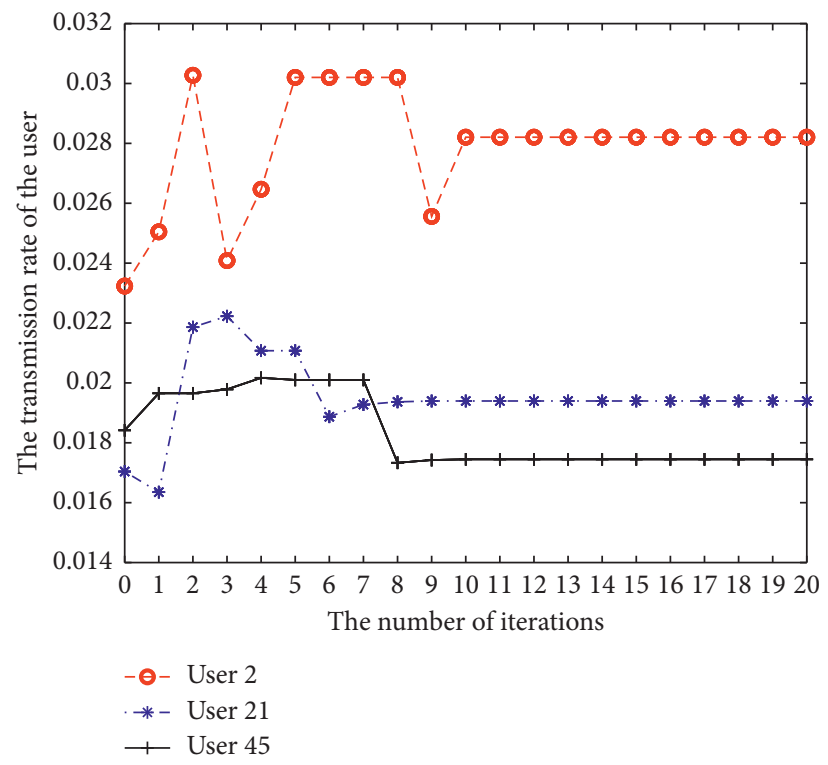

FIgURE 7: The convergence of transmission rate of the UAV users. 


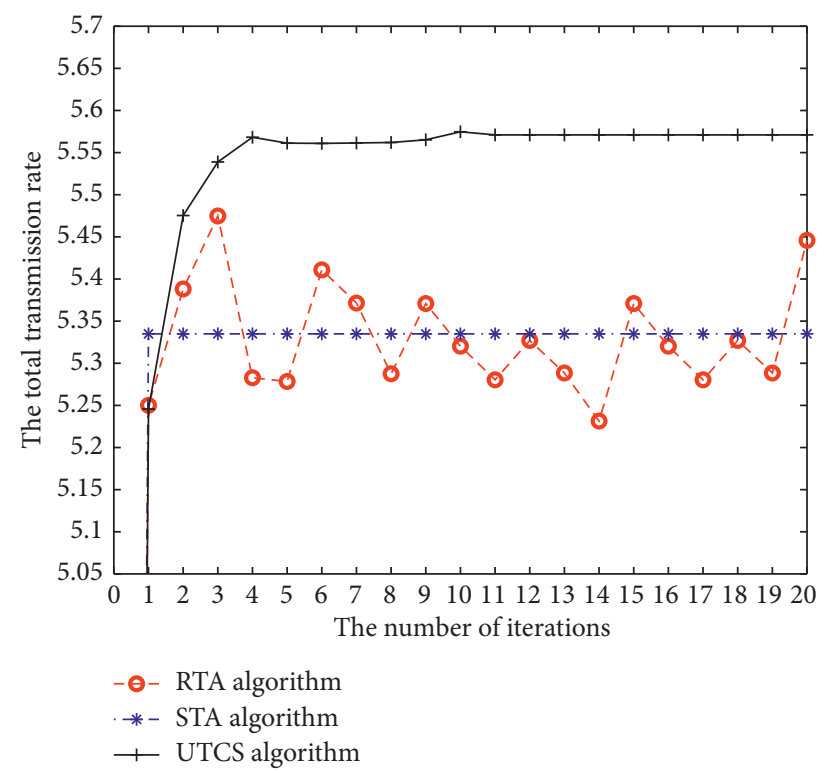

FIgURE 8: Performance comparison of different algorithms in terms of transmission rate for different iterations.

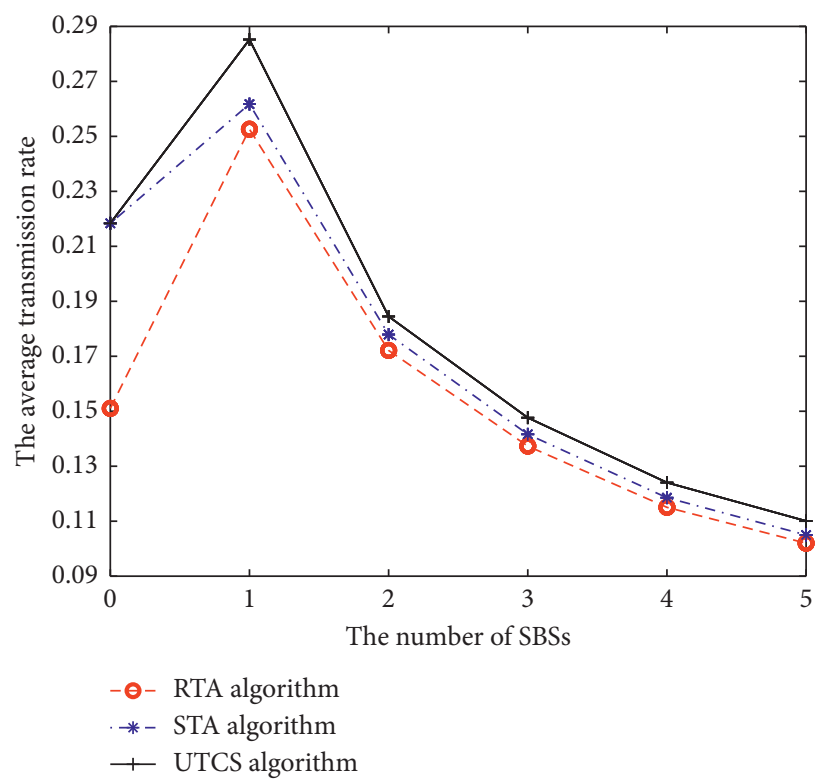

Figure 9: Performance comparison of different algorithms in terms of the total transmission rate for different number of SBSs.

\section{Conclusion}

In this paper, we study the channel selection for $5 \mathrm{G}$ heterogeneous networks to maximize the transmission rate of each user. We apply a noncooperative game method to construct the communication model and prove the existence of NE. A multiple UAV-enabled transmission channel selection (UTCS) algorithm has been proposed to obtain the equilibrium strategy profile of all the UAV users. Experimental results demonstrate that the UTCS algorithm can converge and can perform the best.

\section{Data Availability}

The data used to support the findings of this study are available from the corresponding author upon request. 


\section{Conflicts of Interest}

The authors declare that they have no conflicts of interest.

\section{Acknowledgments}

This work was supported in part by the National Natural Science Foundation of China under Grants 61902029, 61972414, and 61 973161, the Excellent Talents Projects of Beijing under Grant 9111923401, the Scientific Research Project of Beijing Municipal Education Commission under Grant KM202011232015, the Beijing Nova Program under Grant Z201100006820082, the Beijing Natural Science Foundation under Grant 4202066, and the Fundamental Research Funds for Central Universities under Grant 2462018YJRC040.

\section{References}

[1] R. Bi, Q. Liu, J. Ren, and G. Tan, "Utility aware offloading for mobile-edge computing," Tsinghua Science and Technology, vol. 26, no. 2, pp. 239-250, 2021.

[2] D. Kim, J. Son, D. Seo, Y. Kim, and H. Kim, "A novel transparent and auditable fog-assisted cloud storage with compensation mechanism," Tsinghua Science and Technology, vol. 25, no. 1, pp. 28-43, 2020.

[3] Y. Chen, F. Zhao, Y. Lu, and X. Chen, "Dynamic task offloading for mobile edge computing with hybrid energy supply," Tsinghua Science and Technology, 2021.

[4] J. Huang, B. Lv, Y. Wu, Y. Chen, and X. Shen, "Dynamic admission control and resource allocation for mobile edge computing enabled small cell network," IEEE Transactions on Vehicular Technology, p. 1, 2021.

[5] J. Mabrouki, M. Azrour, G. Fattah, D. Dhiba, and S. E. Hajjaji, "Intelligent monitoring system for biogas detection based on the internet of things: mohammedia, Morocco city landfill case," Big Data Mining and Analytics, vol. 4, no. 1, pp. 10-17, 2021.

[6] Y. Chen, F. Zhao, X. Chen, and Y. Wu, "Efficient multi-vehicle task offloading for mobile edge computing in 6G networks," IEEE Transactions on Vehicular Technology, p. 1, 2021.

[7] Y. Zhang, K. Wang, Q. He et al., "Covering-based web service quality prediction via neighborhood-aware matrix factorization," IEEE Transactions on Services Computing, vol. 14, no. 5, pp. 1333-1344, 2021.

[8] X. Zhang, H. Huang, H. Yin, D. O. Wu, G. Min, and Z. Ma, "Resource provisioning in the edge for IoT applications with multilevel services," IEEE Internet of Things Journal, vol. 6, no. 3, pp. 4262-4271, June 2019.

[9] T. Zhao, F. Ye, M. Yan, H. Liu, and S. Basodi, "A survey on algorithms for intelligent computing and smart city applications," Big Data Mining and Analytics, vol. 4, no. 3, pp. 155-172, 2021.

[10] Y. Liu, D. Li, S. Wan et al., "A long short term memory based model for greenhouse climate prediction," International Journal of Intelligent Systems, vol. 37, no. 1, pp. 135-151, 2021.

[11] Y. N. Malek, M. Najib, B. Mohamed, and M. Essaaidi, "Multivariate deep learning approach for electric vehicle speed forecasting," Big Data Mining and Analytics, vol. 4, no. 1, pp. 56-64, 2021.

[12] Y. Zhang, J. Pan, L. Qi, and Q. He, "Privacy-preserving quality prediction for edge-based IoT services," Future Generation Computer Systems, vol. 114, pp. 336-348, 2020.
[13] X. Zhang, H. Chen, Y. Zhao et al., "Improving cloud gaming experience through mobile edge computing," IEEE Wireless Communications, vol. 26, no. 4, pp. 178-183, August 2019.

[14] Y. Chen, N. Zhang, Y. Zhang, X. Chen, W. Wu, and X. S. Shen, "TOFFEE: task offloading and frequency scaling for energy efficiency of mobile devices in mobile edge computing," IEEE Transactions on Cloud Computing, vol. 9, no. 4, pp. 1634-1644, 1 Oct.-Dec.

[15] W. Zhang, X. Chen, and J. Jiang, "A multi-objective optimization method of initial virtual machine fault-tolerant placement for star topological data centers of cloud systems," Tsinghua Science and Technology, vol. 26, no. 1, pp. 95-111, 2021.

[16] L. Qi, X. Wang, X. Xu, W. Dou, and S. Li, "Privacy-Aware cross-platform service recommendation based on enhanced locality-sensitive hashing," IEEE Transactions on Network Science and Engineering, vol. 8, no. 2, pp. 1145-1153, 2021.

[17] S. Mousavi, F. Afghah, J. D. Ashdown, and K. Turck, "Leaderfollower based coalition formation in large-scale UAV network, a quantum evolutionary approach," in Proceedings of the IEEE Conference on Computer Communications Workshops (INFOCOM WKSHPS), Honolulu, HI, USA, April 2018.

[18] J. Li and Y. Han, "A traffic service scheme for delay minimization in multi-layer UAV networks," IEEE Transactions on Vehicular Technology, vol. 67, no. 6, pp. 5500-5504, June 2018.

[19] S. Zhang, H. Zhang, B. Di, and L. Song, "Cellular UAV-to-X communications: design and optimization for multi-UAV networks," IEEE Transactions on Wireless Communications, vol. 18, no. 2, pp. 1346-1359, Feb. 2019.

[20] J. Gu, T. Su, Q. Wang, X. Du, and M. Guizani, "Multiple moving targets surveillance based on a cooperative network for multi-UAV," IEEE Communications Magazine, vol. 56, no. 4, pp. 82-89, April 2018.

[21] A. Bera, S. Misra, and C. Chatterjee, "QoE analysis in cacheenabled multi-UAV networks," IEEE Transactions on Vehicular Technology, vol. 69, no. 6, pp. 6680-6687, 2020.

[22] N. Zhao, X. Pang, Z. Li et al., "Joint trajectory and precoding optimization for UAV-assisted NOMA networks," IEEE Transactions on Communications, vol. 67, no. 5, pp. 37233735, May 2019.

[23] Y. Dai, M. Sheng, J. Liu, N. Cheng, X. Shen, and Q. Yang, "Joint mode selection and resource allocation for $\mathrm{d} 2 \mathrm{~d}$-enabled NOMA cellular networks," IEEE Transactions on Vehicular Technology, vol. 68, no. 7, pp. 6721-6733, July 2019.

[24] M. A. Shattal, A. Wisniewska, B. Khan, A. Al-Fuqaha, and K. Dombrowski, "from channel selection to strategy selection: enhancing VANETs using socially-inspired foraging and deference strategies," IEEE Transactions on Vehicular Technology, vol. 67, no. 9, pp. 8919-8933, Sept. 2018.

[25] H. Ko, J. Lee, and S. Pack, "Joint optimization of channel selection and frame scheduling for coexistence of LTE and WLAN," IEEE Transactions on Vehicular Technology, vol. 67, no. 7, pp. 6481-6491, July 2018.

[26] J. Cui, Y. Liu, and A. Nallanathan, "Multi-agent reinforcement learning-based resource allocation for UAV networks," IEEE Transactions on Wireless Communications, vol. 19, no. 2, pp. 729-743, 2020, Feb. 2020.

[27] D. Liu, Y. Xu, J. Wang et al., "Self-organizing relay selection in UAV communication networks: a matching game perspective," IEEE Wireless Communications, vol. 26, no. 6, pp. 102-110, December 2019.

[28] D. Wu, X. Sun, and N. Ansari, "An FSO-based drone assisted mobile access network for emergency communications," IEEE 
Transactions on Network Science and Engineering, vol. 7, no. 3, pp. 1597-1606, 2020, July-Sept. 12020.

[29] N. Zhang, S. Zhang, J. Zheng, X. Fang, J. W. Mark, and $X$. Shen, "QoE driven decentralized spectrum sharing in 5G networks: potential game approach," IEEE Transactions on Vehicular Technology, vol. 66, no. 9, pp. 7797-7808, 2017. 